\title{
COORDINATED WALKING OF STICK INSECTS ON A MERCURY SURFACE
}

\author{
D. GRAHAM AND H. CRUSE \\ FB Biologie Universität Kaiserslautern, \\ D-6750 Kaiserslautern, Federal Republic of Germany
}

(Received 28 August 1980)

\begin{abstract}
SUMMARY
Adult stick insects walk on a mercury surface at step frequencies in the range $\mathrm{I}-4 \mathrm{~Hz}$ with a coordination similar to that found in free-walking adults at their maximum step frequency of $3 \mathrm{~Hz}$. The amplitude of leg movement covers the same range as that found in free-walking animals. The use of a mercury substrate effectively removes mechanical interactions between the legs, showing that such influences cannot be the cause of the coordinated behaviour observed in this experimental situation. The dependence of the maximum step frequency upon the resistance to retraction provided by the substrate suggests that feedback from the periphery determines the step period. The variation in the anterior extreme position of a leg is less than that of the posterior extreme position, indicating that the latter may be more strongly influenced by coordinating interactions between the legs.
\end{abstract}

\section{INTRODUCTION}

The central versus peripheral basis of inter-leg coordination in walking animals has been a stimulating controversy in the field of locomotion for many years, and has motivated many experimental scientists to attempt to exclude various fields of sensory input and search for changes in leg coordination that would clarify the role of central and peripheral components in walking. Wendler (1964) established that the basic pattern of coordination in walking is still present when the animal's body is supported rigidly above a wheel mounted on a fixed axle. This experiment effectively disproved earlier suggestions that changes in loading of some legs, by others, might induce such patterns. It was then suggested that perhaps mechanical influences through the substrate could produce such coordinated patterns of movement. This hypothesis has been more difficult to test. However, the recent construction of a lightweight, counter-balanced double treadwheel has shown that in the stick insect right and left sides remain coupled through the central nervous system in the absence of any mechanical coupling through the substrate (Graham, I981 $a$ ).

Recent work by Cruse (1976) and Graham ( $1981 b$ ) suggests that substrate coupling between the legs on the same side does provide a mechanical pathway for force interactions to occur between the legs. The present work tests the hypothesis that ch substrate influences might provide the coupling between legs responsible for me normal walking coordination. 

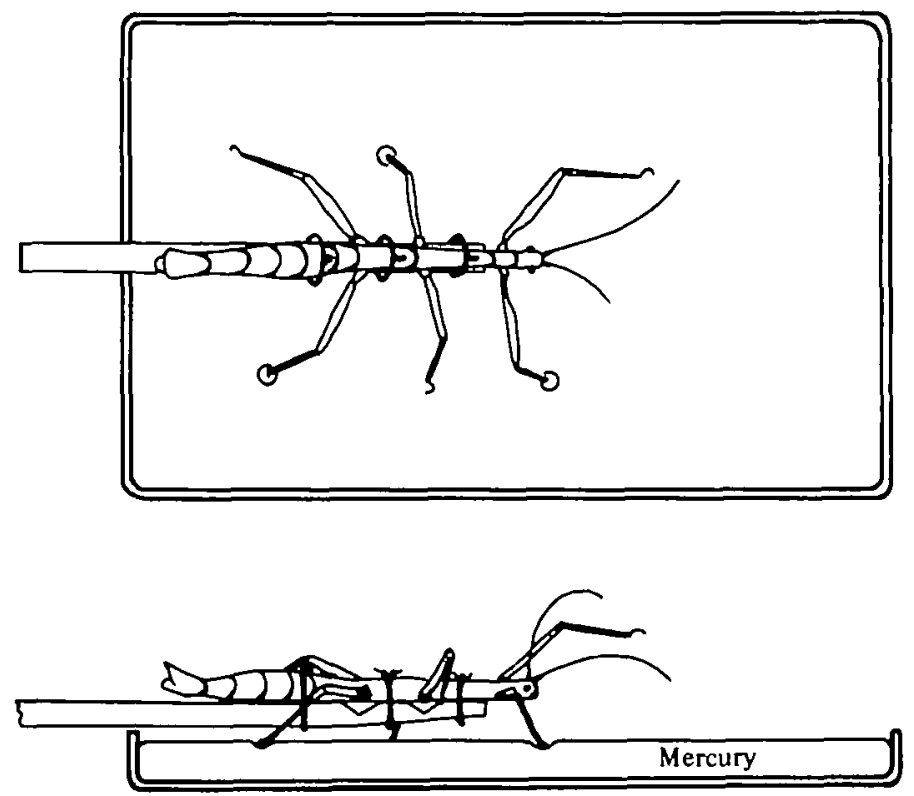

(b)

$\mathrm{R}{ }^{3} \cdots \cdots \cdots \cdots$

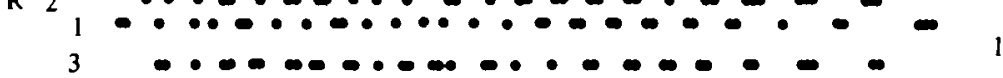

$\mathrm{L}_{2}{ }_{1}^{3} \cdots$

R $2_{1}^{3}$

$\mathrm{L}^{3}{ }_{1}^{3}$

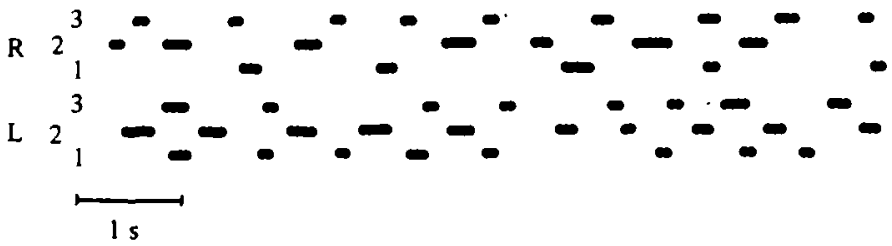

Fig. I. Experimental arrangement for walking on mercury surface (a), and (b) the step patterns for scenes 1,5 and 9. Filled circles show film frames during which the leg is not in contact with the mercury surface. 


\section{METHODS}

Adult female stick insects (Carausius morosus) were used. The insect was attached ventrally to a rigid balsa stick using wire clips to hold the meso- and metathorax and the anterior part of the abdomen (Fig. I $a$ ). The ventral surface was $8 \mathrm{~mm}$ above a clean mercury surface $10 \mathrm{~mm}$ deep. The standing or walking animal exerted vertical forces downward on the liquid without breaking through the surface. This produced small circular depressions which reflected the oblique illumination used during filming. The walking animal was filmed at 18 frames $\mathrm{s}^{-1}$ from above using an S-8 Beaulieu movie camera.

Legs are represented by $L$ or $R$ for left and right and numbered $I-3$ from front to rear. Each step consists of two parts; either the leg is lifted from the surface or it is in contact with the mercury. When not in contact with the mercury the leg is moving forward in a recovery stroke which places the leg in an anterior position to prepare for the next movement to the rear. This is called a protraction (swing phase or return stroke). When the leg is on the substrate it depresses the mercury surface and it can retract (stance phase or power stroke). In a free-walking animal the leg may be actively moved backwards or may be moved by the other legs (Cruse, 1976; Graham, 1981 $b$ ). On mercury only the former possibility exists.

In the selected scenes, the following measurements were made. The onset of protraction and retraction were used as convenient time marks for the evaluation of step period, protraction duration, and the lag between the protraction of different legs. Lag is represented by $R_{3} L_{R_{1}}$, which denotes the interval between protraction in leg $\mathrm{R}_{3}$ and the subsequent metachronal protraction of RI. The period is defined as the sum of protraction time and retraction time. Phase was calculated as the lag between the reference leg and the given leg, divided by the period of the reference leg. The phase of LI referenced to L2 is written LI:L2.

The position of the tarsus was measured for each film frame and the extreme anterior (AEP) and posterior position (PEP) were determined for each leg. The extreme positions are defined respectively as the position at which the protracting leg touches the surface and the position at which the leg releases contact with the surface. Both were measured as a projection on the longitudinal axis of the body. The origin of this longitudinal coordinate axis is the anterior tip of the head (following Bässler, 1972). Points in front of the head are positive. The mean body length of the animals was $73 \mathrm{~mm}$. For details of body measurements see Cruse (1976).

Step amplitude was calculated from the extreme positions of the tarsus and is defined as AEP minus PEP. Note that this quantity is not the same as that which is determined in a free-walking animal from the separation of footprints and which includes a contribution from body displacement during the protraction stroke.

\section{RESULTS}

A liquid surface, that does not wet the leg, acts as a flexible membrane producing an upthrust equal to the weight of mercury displaced. The legs produced depressions - the surface corresponding to a volume of $\sim 4 \mathrm{~mm}^{3}$ giving an upthrust of $\mathrm{I} \cdot 2 \mathrm{mN}$ (12 pd). This is similar to the weight normally carried by the leg of a standing 
animal. If the depression in the surface moves horizontally it does so by shearif the surface layer against the molecules below. The shear forces producing flow in a liquid are very small and that required to move the leg is correspondingly low.

Attempts to measure the force required to move an insect leg across the mercury surface were unsuccessful due to the lack of a sufficiently sensitive force transducer.

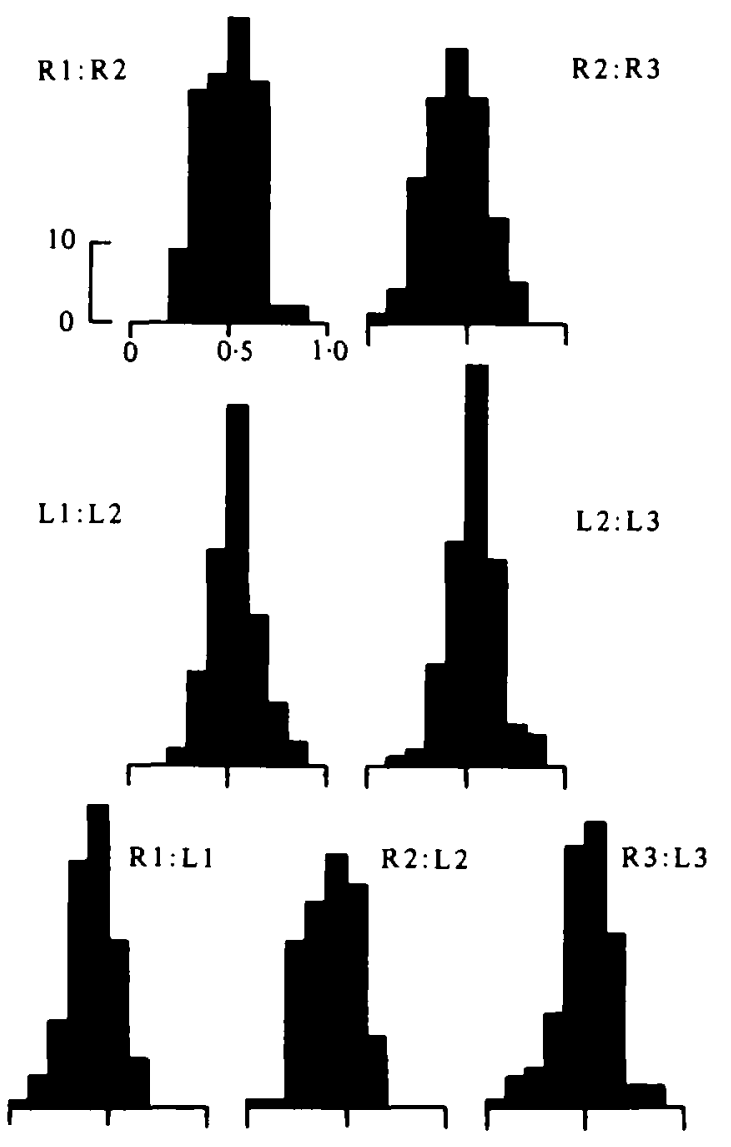

Fig. 2. Phase histograms of leg protractions for all 9 scenes.

The measurements did show that the force required was $<$ 10 $\mu \mathrm{N}(\mathrm{I} \mathrm{mp})$ at velocities up to $20 \mathrm{~cm} \mathrm{~s}^{-1}$. This is approximately $0.1 \%$ of the force required to accelerate the body during normal walking.

When stimulated by gently brushing the abdomen, the animals walked with clear and precise movements similar to those used by free walking animals (Fig. I $b$ ). Films were taken of 4 animals, and in all walks the legs moved forwards and backwards rhythmically.

All of the legs usually showed well-coordinated movements for 10-35 successive steps of each leg. From film of over 800 steps of an individual leg a sample of 383 steps was selected, on the basis of all legs appearing to be active together, and was evaluated in the form of step plots. Approximately $80 \%$ of the middle leg stem examined formed part of a metachronal sequence consisting of hind, middle an 


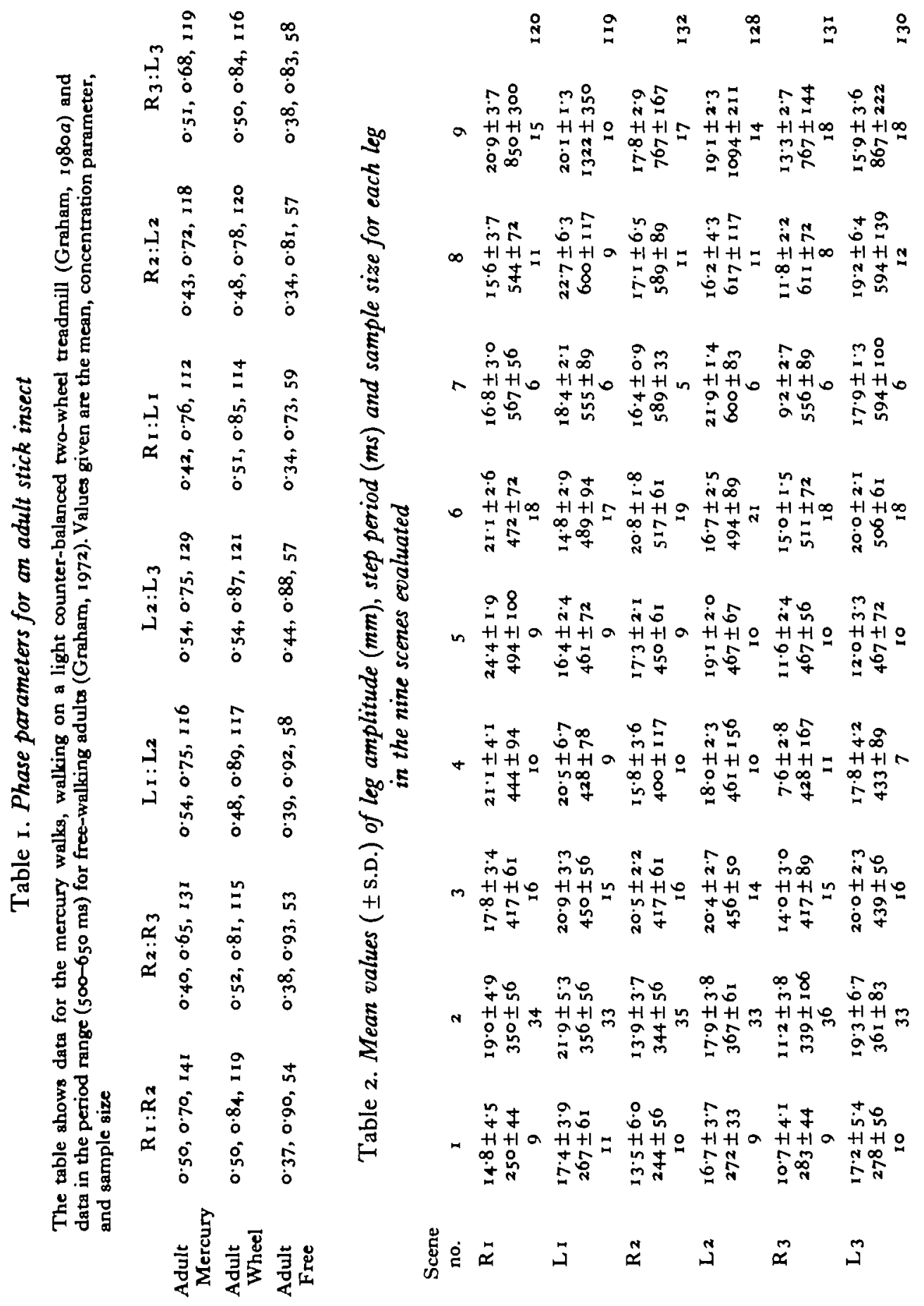


front legs moving quickly forward above the mercury surface separated by approx. mately equal time intervals. Nine scenes from 3 animals with a total of 154 steps of each leg were examined in greater detail. These scenes were selected as typical of coordinated walking in these animals at stepping frequencies ranging from 0.8 to ${ }_{4} \mathrm{~Hz}$ and were ordered in ascending period.

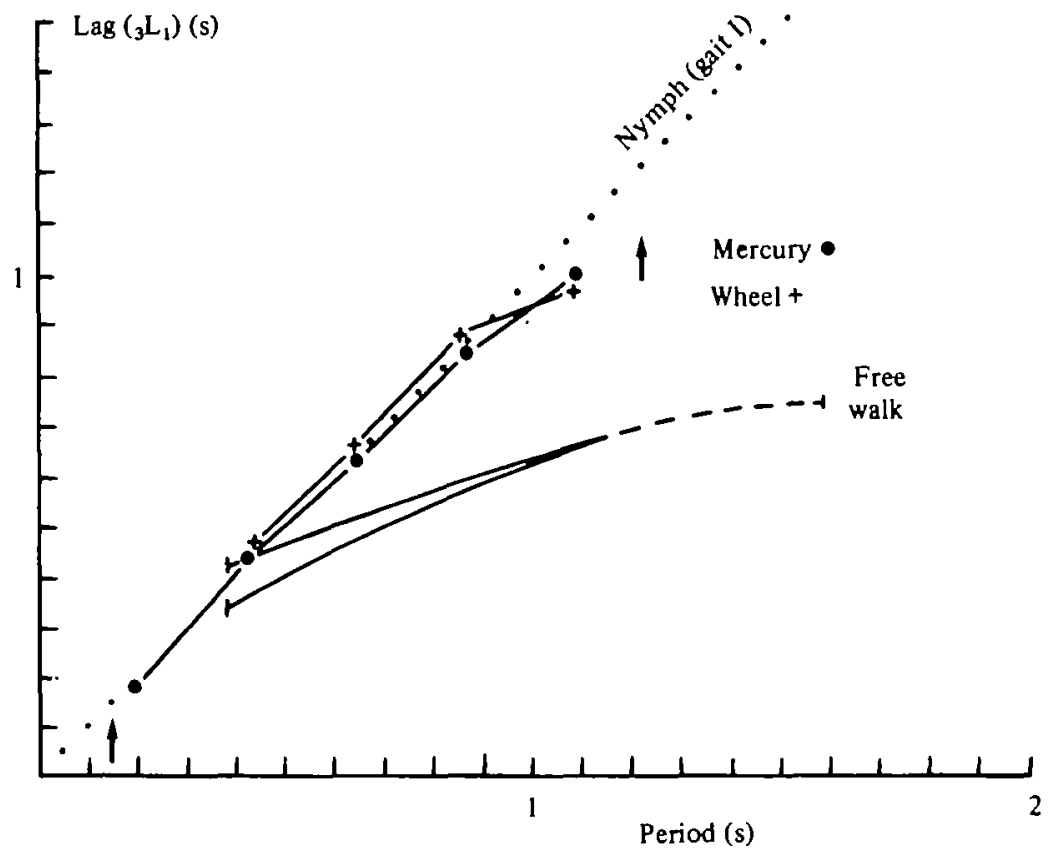

Fig. 3. Lag $\left(\mathrm{s}_{3}\right)$ versus period relation for 1 st instar nymphs walking free and adults walking free, on mercury and on wheels (Graham, D. I $980 a, 1972$ ). The data range for all 9 scenes is shown by the vertical arrows.

\section{COORDINATION}

As no systematic differences between the phase values for individual scenes were found, all data are presented together in Fig. 2. The mean phase and concentration parameter (Batschelet, 1965) are given in Table I. Comparing these values with corresponding values obtained from animals walking free (Graham, 1972) and walking on a treadwheel (Graham, I980a) only minor quantitative differences were found. Thus the fundamental temporal coordination between the legs is the same in animals with and without mechanical coupling between the legs. The lower values of the concentration parameters do not necessarily mean that the exactness of timing is worse in the mercury experiments, but rather that they represent a slightly less critical selection procedure than is sometimes adopted in studies of walking coordination.

Fig. 3 shows the lag $\left({ }_{3} L_{1}\right)$ as a function of period. The lag is strongly dependent upon period and the behaviour is similar to that found in Ist instar stick insects (gait I) and for adults walking on light wheels (Graham, 1980a). Fig. 4 showa protraction duration as a function of period and the data are highly correlated, wit] 


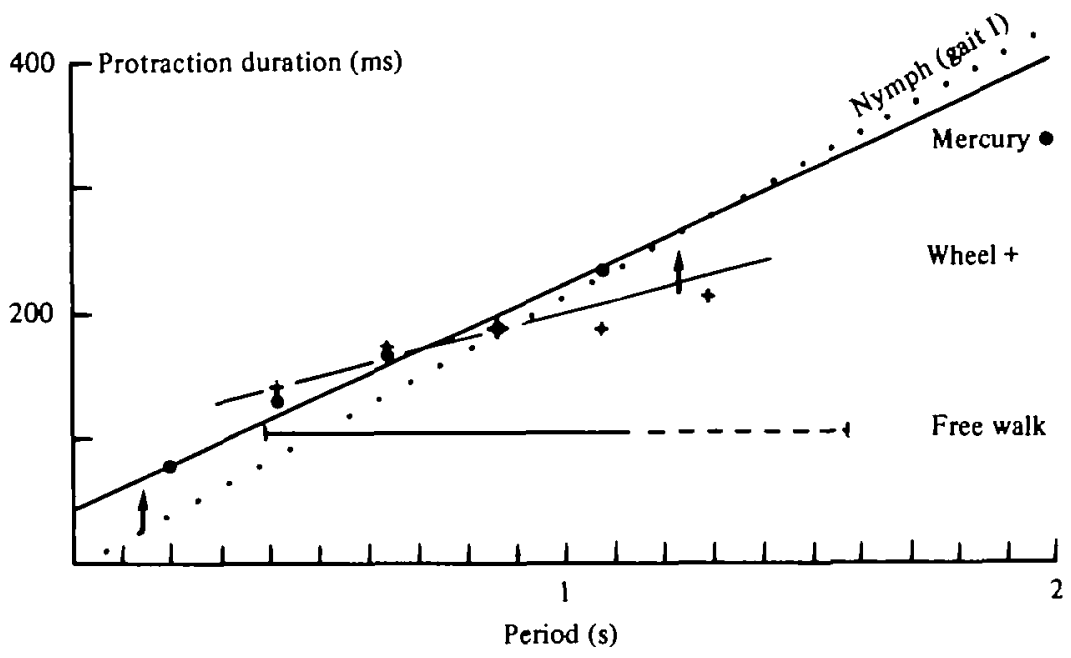

Fig. 4. Protraction duration as a function of period for the conditions described in Fig. 3 . The correlation coefficient for the mercury walks was 0.643 and the regression line is protraction duration $=44.4+0.18 \times$ period.
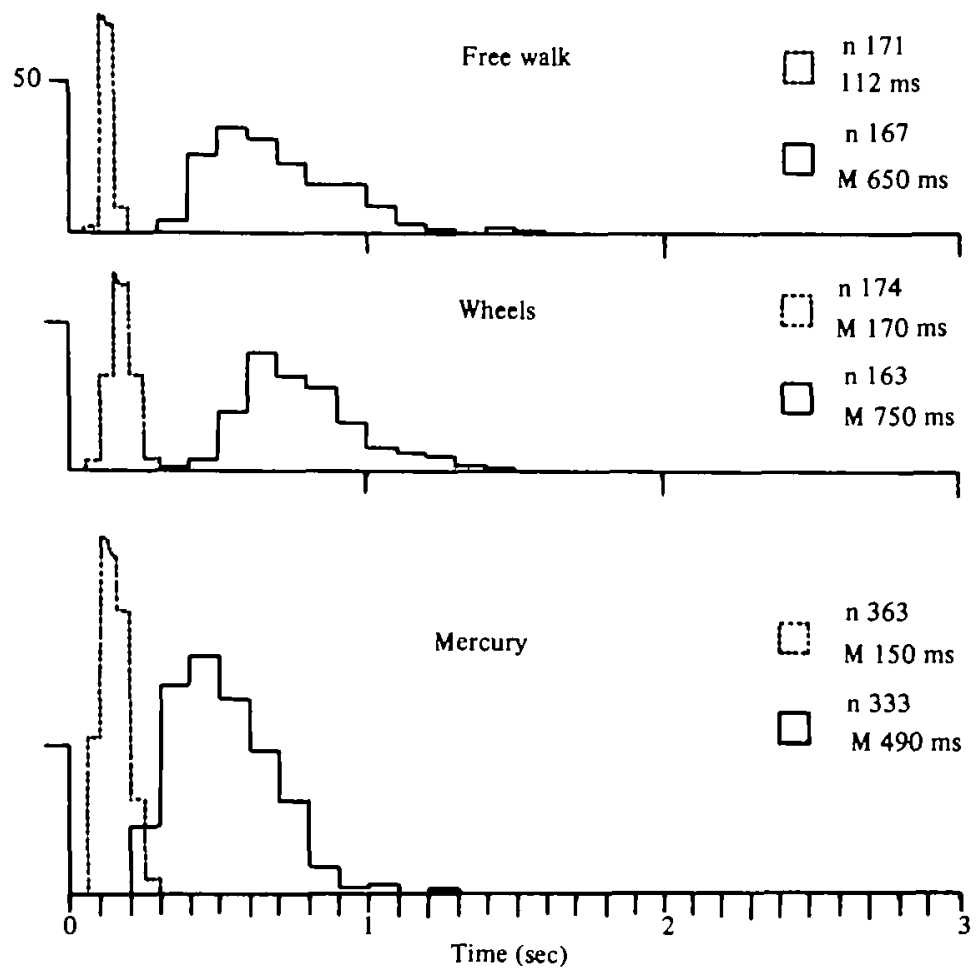

Fig. 5. Distribution of protraction duration (dotted lines) and step period (continued lines) for adult stick insects walking free, on wheels, and supported above mercury. 
a slope (regression with period as independent variable) of 0.18 . This is close that for the Ist instar gait 0.23 (Graham, I972).

\section{TIMING OF LEG MOVEMENTS}

In walks on mercury, the mean period of $490 \mathrm{~ms}$ is shorter than that for freewalking stick insects $(650 \mathrm{~ms})$. This difference is highly significant, and approximately $30 \%$ of the steps recorded on mercury had periods of less than $400 \mathrm{~ms}$, while $98 \%$ of the free-walk steps had periods greater than this value. In all the walks analysed for free-walking animals and those on light wheels, no walks with periods of less than $380 \mathrm{~ms}$ have been reported. The protraction duration and leg amplitude are similar to those of free-walking animals and in consequence the short step period must be attributed to a higher mean velocity during the retraction movement of the leg.

Values of step period for the legs along the body axis do not differ significantly from each other (Table 2) but legs of the left side sometimes walk with higher frequency than those of the right side of the body, and in these cases the contralateral legs show gliding coordination as described by Graham (1972) during turning behaviour. At such times the hind leg on the outside of the turn showed strong tibial flexion during protraction and extension in retraction, rather than rotating as a whole about the coxo-thoracic joint. This behaviour can sometimes appear in the front leg on the inside of a turn and produces steps in both instances with a rather smaller amplitude than normal (Table 2). Most steps, however, showed both rotation and tibial extension.

\section{LEG POSITION}

The mean values of the amplitude of leg movement are shown for the different legs and different walks in Table 2 together with their standard deviations. It can be seen that within one walk the amplitude of different legs can sometimes differ by as much as a factor of 2 , but this is associated with the lack of leg rotation already described and is an indication that the animal is turning. The temporal coordination did not always alter with these changes in leg amplitude, and no differences could be seen between the corresponding phase plots of the individual scenes. The standard deviations of the period are similar to those shown for leg amplitude, and differences are not significant.

The mean values of the anterior extreme positions (AEP) and of the posterior extreme positions (PEP) are shown for each leg in Table 3. The values of the standard deviations are frequently smaller for the AEP than for the PEP. In the front legs this is true in 17 out of 18 walks (right and left for 9 scenes), in the middle legs in 13 out of 18 walks, and in the hind legs in 12 out of 18 cases. As the AEP of middle and hind legs is influenced by the PEP of the leg in front (Cruse, 1979a; H. Cruse \& F. Krieger, in preparation), it is to be expected that this difference would be less marked in the middle and hind legs. Collecting all values of all scenes one obtains a variance ratio $\sigma_{\mathrm{PEP}}^{2} / \sigma_{\triangle \mathrm{EP}}^{8}$ of $0.15(n=75)$ for the leg $\mathrm{LI}$ and of 0.35 $(n=85)$ for the leg RI. This shows that for the front legs the anterior extreme position is much more constant relative to the body than is the posterior extrem position. 
Table 4 compares the mercury leg positions with the results of Bässler (1972) and Cruse (1976) and shows that the range of the mean values is similar to that for animals walking free on a horizontal plane, with the one exception that the PEP of the hind legs walking on mercury lies somewhat in front of that for the freewalking animals.

Table 3. Mean values ( \pm S.D.) of the anterior (AEP) and pasterior (PEP) extreme position of the legs, as described in the text

\begin{tabular}{rrr} 
& \multicolumn{1}{c}{ Left } & \multicolumn{1}{c}{ Right } \\
Front leg AEP & $16.1 \pm 2.1$ & $14.2 \pm 2.9$ \\
& $n=122$ & $n=125$ \\
PEP & $-3.5 \pm 4.5$ & $-5.1 \pm 4.7$ \\
& $n=121$ & $n=125$ \\
Middle leg AEP & $-16.0 \pm 2.4$ & $-13.9 \pm 2.4$ \\
& $n=129$ & $n=134$ \\
PEP & $-33.9 \pm 3.5$ & $-31.1 \pm 4.3$ \\
& $n=129$ & $n=134$ \\
Hind leg AEP & $-35.9 \pm 3.8$ & $-36.5 \pm 4.7$ \\
& $n=125$ & $n=133$ \\
PEP & $-54.7 \pm 4.1$ & $-48.5 \pm 5.1$ \\
& $n=130$ & $n=132$
\end{tabular}

Table 4. Range of the mean values of AEP and PEP on mercury compared with those of Bässler (1972) and the means ( \pm S.D.) obtained by Cruse (1976) for free-2oalking animals

\begin{tabular}{|c|c|c|c|}
\hline & Walk on mercury & $\begin{array}{c}\text { Free walking } \\
\text { (Blssler, 1972) }\end{array}$ & $\begin{array}{l}\text { Free walking } \\
\text { (Cruse, 1976) }\end{array}$ \\
\hline $\begin{array}{c}\text { Front legs } \\
\text { AEP } \\
\text { PEP }\end{array}$ & $\begin{array}{l}11 \cdot 1-17.8 \\
0.1--10.1\end{array}$ & $\begin{array}{l}13 \cdot 4-18 \cdot 3 \\
-1 \cdot 2--11 \cdot 0\end{array}$ & $\begin{array}{r}11 \pm 3 \\
-7 \pm 4\end{array}$ \\
\hline $\begin{array}{c}\text { Middle leg } \\
\text { AEP } \\
\text { PEP }\end{array}$ & $\begin{array}{l}-12.3-18.5 \\
-27.5-37.5\end{array}$ & $\begin{array}{l}-10.9--17.5 \\
-32.8--38.5\end{array}$ & $\begin{array}{l}-17 \pm 2 \\
-35 \pm 4\end{array}$ \\
\hline $\begin{array}{c}\text { Hind legs } \\
\text { AEP } \\
\text { PEP }\end{array}$ & $\begin{array}{l}-31 \cdot 5-43.5 \\
-42 \cdot 7-57 \cdot 4\end{array}$ & $\begin{array}{l}-32 \cdot 9-45 \cdot 1 \\
-55 \cdot 0-65 \cdot 3\end{array}$ & $\begin{array}{l}-40 \pm 3 \\
-58 \pm 4\end{array}$ \\
\hline
\end{tabular}

\section{VELOCITY OF LEG MOVEMENT}

The retraction velocity of each leg between consecutive frames $(55.6 \mathrm{~ms})$ was calculated for each scene and each leg. The velocity was plotted against the position of the leg in the retraction stroke (Fig. $6 a$ ) and against time. These velocity profiles are similar for each leg. In Fig. 6 for a middle leg, the plots of position as abscissa (Fig. $6 a$ ) are obviously more concise than those plots using time as the abscissa (Fig. $6 b$ ). The reason for this is that the leg can either pause at the beginning of the retraction period for $>50 \mathrm{~ms}$ or it can start almost immediately with a retraction movement. In the position plot (Fig. $6 a$ ), this produces superposition of a number of points on the origin. To show this more clearly some individual steps from the time plot of Fig. $6 b$ are repeated separately in Fig. $6 c$, with the points beThing to the same step connected. This shows that the corresponding effect of 


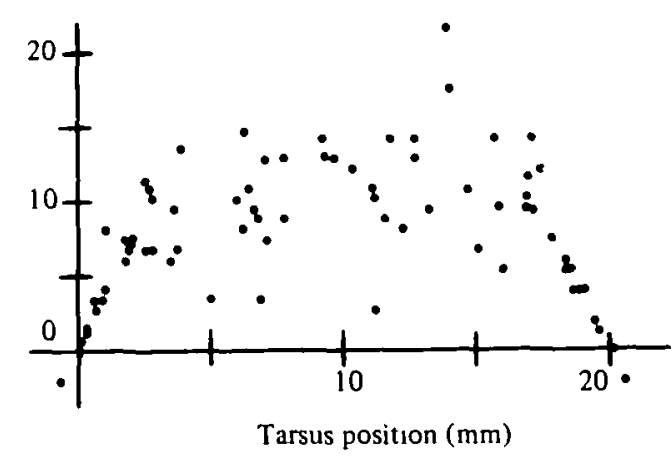

(a)
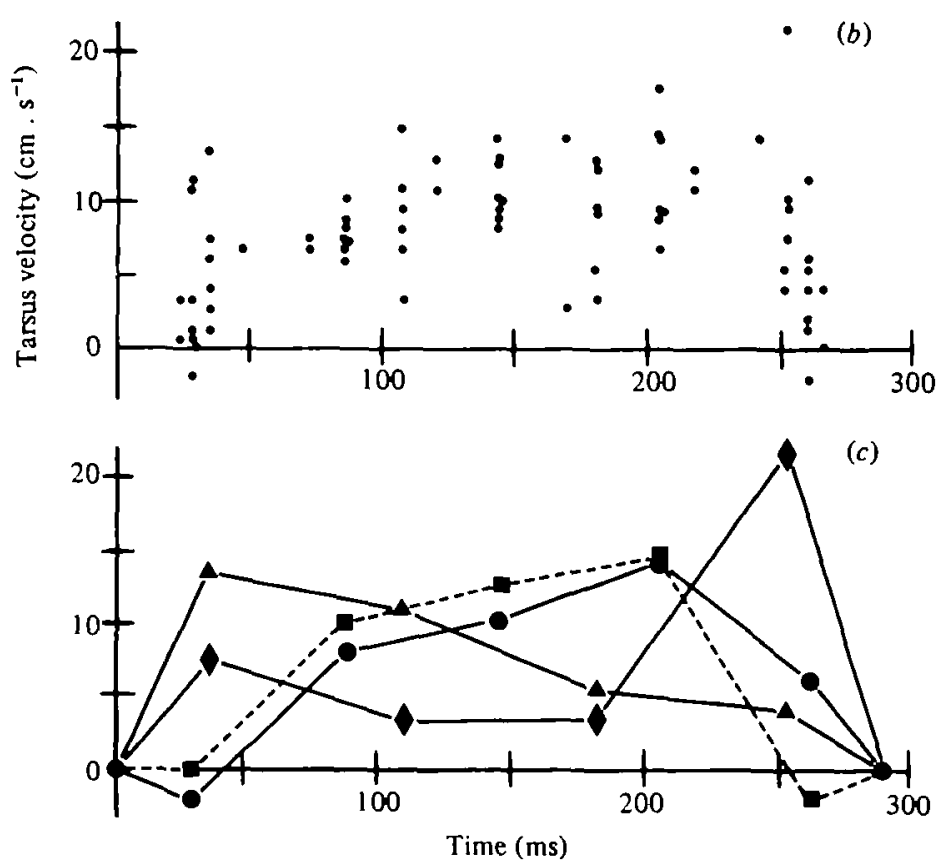

Fig. 6. Velocity of a left middle leg a a function of (a) position relative to the body and (b) time. Both are normalized to the mean values shown in scene 3 . In $(c)$ the velocity points corresponding to single cycles $(\square \Delta \wedge)$ are linked by lines.

a leg not moving can also occur at the end of the retraction stroke before starting the protraction movement. No differences were observed concerning the duration of standing at the beginning or at the end of the retraction stroke or between different legs, but such pauses of $c a .50 \mathrm{~ms}$ are typical of walking on mercury. Both effects are particularly marked in slow-walking animals. At higher or lower step frequency than the example of Fig. 6 the low film rate in one case and the slow rate of movement in the other make it difficult to calculate the exact duration of these pauses, but they can be estimated to lie in the range of $30-100 \mathrm{~ms}$.

The average data used in Fig. $6 a$ might suggest that the individual leg is relatively uniform in its behaviour and accelerates during the first part of the stroke, maintains a constant velocity, and then decelerates rapidly before reaching the PEP, but thi is not the case. The individual records in Fig. $6 c$ were chosen to demonstrate thw 
Dariability from step to step. This variability also exists in the forces applied by the legs during the retraction phase. The only strongly coordinated feature of the mercury walks is the time of lifting the legs in protraction. The retraction force is not strongly coordinated, and maxima in the retraction velocity occur arbitrarily at different times during the stance phase in the three legs of a retracting tripod.

\section{DISCUSSION}

The mercury surface forms a supporting cup around the tarsus stimulating the tarsal hairs and other sense organs measuring contact and joint displacement. However, each leg is effectively isolated from the forces applied by other legs. The leg also experiences an upthrust as in normal surface walking, but this is again completely independent of the vertical forces exerted by the other legs. The internal anatomy of the stick insect shows that there are no mechanical connexions between the legs, and therefore each leg is entirely independent of the others unless they touch one another. No such contacts were observed in the film record.

The first observation is that the insect can stand still and is not in a continuous state of slipping. Therefore there must be an equilibrium position for the legs when standing, in which no reaction is expected and against which no continuously maintained force need be applied.

When the animal is stimulated the legs move with regular and fully coordinated movements. The periodicity of each leg does not differ significantly from any other leg despite significant changes in amplitude of some legs which appear to be attempts to turn the body. However, the average is significantly shorter than that for freewalking and wheel-walking animals under similar stimulation conditions. The protraction duration is similar to that for wheel-walking animals, therefore it is the retraction duration which is reduced to a value comparable with the protraction duration at the maximum step frequency. Thus the retraction velocity appears to depend heavily on the resistance to motion and determines the maximum step frequency of the walking system.

The coordination of the animal is identical to that of the Ist instar stick insect using gait $I$ in a free walk, and the slope and intercept of protraction duration versus period do not differ significantly from those of ist instar walking. The behaviour is also similar to that found for adults walking on light wheels and decerebrate adults walking free (Graham, 1979).

The leg amplitudes for all legs and both AEP and PEP positions for the front and middle legs are similar to those of free-walking or wheel-walking animals. The right rear leg has an average PEP which tends to be further forward, but this is caused by the smaller step amplitude of this leg associated with turning in some scenes.

An earlier paper of Cruse (1979a) shows that the hind legs tend to place the leg (AEP) close to the tarsus of the middle leg. A similar effect was found in the mercury walks, where the differences between the variance of AEP and PEP are significant $(F$ test, $P<0.1 \%)$ for the front and middle legs but not for the hind legs $(P>10 \%)$. For the front and middle legs, the AEP is relatively constant but PEP has twice me standard deviation of AEP. For the hind legs, the AEP follows the PEP of the 
leg in front, giving a similar standard deviation for both AEP and PEP in thm case. This increased variability of PEP in the front and middle legs may be the result of coordinating signals from other legs. The PEP appears to be a particularly appropriate point at which to alter the relative phase of the leg, as the system must decide at this moment if lifting of a leg is appropriate or not in the context of the support available from other adjacent legs.

Another feature of AEP and PEP in the mercury-walking animals is that the leg tends to pause at these points in the leg cycle for intervals ranging from 30 to $100 \mathrm{~ms}$. A similar effect has been observed in decerebrate stick insects (Graham, 1979), and locusts are often seen to elevate the leg slightly above the surface before protraction when walking slowly (Burns \& Usherwood, 1973).

In free-walking adult stick insects these pauses are obscured by the body displacements produced by other legs. In wheel-walking and mercury-walking adults, free-walking decerebrates, and rst instar nymphs, the use of a tripod gait at all step frequencies makes this momentary pause at AEP and PEP easily detectable at both fast and slow speeds of walking. It is clear that when the leg is first placed in contact with the ground it often remains stationary for $30-100 \mathrm{~ms}$ before moving to the rear in the propulsion stroke.

In conclusion, the present results show that adult stick insects walk on a mercury surface with fully coordinated leg movements similar to those of a ist instar nymph or an adult walking on a light wheel. This observation must reject any hypothesis which suggests that leg coordination is produced by leg interactions through the substrate. The additional observation that the maximum rate of stepping is significantly increased when the resistance to retraction is reduced is compatible with the 'peripheral oscillator' model of Graham (1977) and the model of Cruse (1979b, 1980), which are based upon the transfer of position information between the legs. This result rejects the 'central oscillator' version of Graham (1977) or any exclusively endogenous model in which individual leg movements follow a central oscillator which determines the timing of the leg cycle. Such systems would be expected to show an increase in leg amplitude but no change in the period when the resistance to retraction is decreased.

This work was supported by Deutsche Forschungsgemeinschaft, $\mathrm{Ba} 578$ and Cr 58. The authors would like to dedicate this article to their indefatigable typist Frau Herberich, whose help and concern have been invaluable.

\section{REFEREN CES}

BXssler, U. (1972). Zur Beeinflussung der Bewegungsweise eines Beines von Carausius morosus durch Amputation anderer Beine. Kybernetik 10, r 10-1 14.

BAtschelet, E. (1965). Statistical methods for the analysis of problems in animal orientation and certain biological rhythms. A.I.B.S. monograph.

Burns, M. D. \& Usherwood, P. N. R. (1973). Control of walking in orthoptera. I. Leg movements in normal walking. Y. exp. Biol. 58, 45-58.

Cruse, H. (1976). The function of the legs in the free walking stick insect, Caransius morosus. $\%$. comp. Physiol. I12, 235-262.

Cruse, H. (1979a). The control of the anterior extreme position of the hindleg of a walking insech Carausius morosus. Physiol. Entomal. 4, I21-124. 
DUSE, H. (1979b). A new model describing the coordination pattern of the legs of a walking stick insect. Biol. Cybernetics 32, 107-1 13.

Cruse, H. (1980). A quantitative model of walking incorporating central and peripheral influences. I and II. Biol. Cybernetics 37, 131-144.

Graham, D. (1972). Analysis of walking movements in $18 t$ instar and adult stick insects (Carausius morosus). Y. comp. Physiol. 81, 23-52.

Graнam, D. (1977). Simulation of a model for the coordination of leg movement in free walking insects. Biol. Cybernetics 26, 187-198.

Graham, D. (1979). Effects of circum-oesophageal lesion on the behaviour of the stick insect Carausius morosus. I and II. Biol. Cybernetics 32, 139-145 and $147-152$.

Graham, D. ( $198 \mathrm{r} a$ ). Walking kinetica of the stick insect using a low inertia, counter-balanced pair of independent treadwheels. Biol. Cybernetics (In the Press.)

Graham, D. ( $198 \mathrm{r} b)$. 'Lurching' locomotion in insects. 9 . exp. Biol. (submitted).

Wendlar, G. (1964). Laufen und Stehen der Stabheuschrecke Carausius morosus. Z. vergl. Physiol. 48, 198-250. 
